\title{
Improved robustness in the junction region for postoperative breast cancer including supraclavicular nodes using hybrid VMAT
}

Hideharu Miura ( $\nabla$ miura@hiprac.jp )

Hiroshima High-Precision Radiotherapy Cancer Center

\section{Yoshiko Doi}

Hiroshima High-Precision Radiotherapy Cancer Center

Minoru Nakao

HIPRAC

Shuichi Ozawa

HIPRAC

Masahiko Kenjo

HIPRAC

Yasushi Nagata

HIPRAC

\section{Research Article}

Keywords: Breast cancer, Hybrid-VMAT, junction region, patient motion, plan robustness

Posted Date: February 23rd, 2021

DOl: https://doi.org/10.21203/rs.3.rs-220715/v1

License: (9) (i) This work is licensed under a Creative Commons Attribution 4.0 International License.

Read Full License 


\section{Abstract \\ Purpose}

To improve the hybrid VMAT treatment plan robustness for postoperative breast cancer patients considering small patient motion shifts during treatment using a 3D-CRT with a dose gradient in the junction region.

\section{Methods}

Locoregional supraclavicular nodes of breast cancer patients were planned using 3D-CRT and VMAT. A 3D-CRT plan with a dose gradient on the cranial side was applied by shifting the jaw to reduce hot or cold spots. The VMAT plan was optimized based on the results of the 3D-CRT plan calculation. Hybrid plans were created by the sum of the 3D-CRT and VMAT plans. To simulate patient motion, the plans were recalculated with the VMAT plan simulating isocenter superiorly (separation) or inferiorly (overlap) shifted by 1,2 , and $3 \mathrm{~mm}$. The shifted plans were compared with the non-shifted plans considering the clinical target volume (CTV) $\left(\mathrm{D}_{98 \%}\right.$ or $\left.\mathrm{D}_{2 \%}\right)$.

\section{Results}

The $\mathrm{D}_{2 \%}$ value of the CTV with perfectly aligned fields for the hybrid VMAT plan with high- or low-dose gradients on the 3D-CRT plan increased from $102.8 \% / 102.9-107.2 \% / 105.7 \%, 114.9 \% / 110.9 \%$, and $122.2 \% / 115.5 \%$ for each $1 \mathrm{~mm}, 2 \mathrm{~mm}$, and $3 \mathrm{~mm}$ overlapped shift, respectively. The value of $D_{98 \%}$ to the CTV with perfectly aligned fields decreased from $95.7 \% / 95.6-90.0 \% / 93.1 \%, 81.2 \% / 88.4 \%$, and $72.8 \% / 83.5 \%$ for each $1 \mathrm{~mm}, 2 \mathrm{~mm}$, and $3 \mathrm{~mm}$ separated shift, respectively.

\section{Conclusions}

By employing a 3D-CRT plan with a low dose gradient on the cranial side, the dose differences can be decreased. A more robust treatment delivery option can be achieved for breast cancer treatment using our proposed hybrid VMAT.

\section{Introduction}

Breast cancer accounts for a significant proportion of female patients who are treated with radiation therapy (RT). Surgery, systemic therapy, and RT are used for breast cancer treatment. Adjuvant RT following lumpectomy or mastectomy is performed to reduce locoregional recurrence and improve survival [1-3]. Traditionally, two opposing tangential and anterior fields are mainly used for the chest wall area and supraclavicular nodes, respectively [4-7]. The anterior field should be precisely matched with 
the two tangential fields to avoid hot and cold spots in the junction region. Several approaches have been proposed to improve inhomogeneities in the junction region [7-18]. Intensity-modulated radiation therapy (IMRT) or volumetric-modulated arc therapy (VMAT) for breast cancer treatment have been proposed to provide better dosimetric results and to improve dose conformity to the target [9-18]. Most hybrid VMAT planning studies for breast treatment have used either IMRT or VMAT, which uses open tangential threedimensional conformal radiotherapy (3D-CRT) fields with main dose weighting as a base dose plan [914]. We used a combination of two tangential fields with unequal cranial field edges above the breast and VMAT fields to obtain a significant improvement in homogeneity and conformity for the targets, which is called "Hybrid VMAT" [18]. Our method differs from the one commonly referred to as the hybrid VMAT technique. There is no irradiation to the contralateral chest wall area and a low dose is given to the lung because our hybrid VMAT method uses only the conventional tangential field for the chest wall area. This method is less susceptible to respiratory motion and breast deformation [19]. Studies analyzing IMRT or VMAT have used a virtual bolus outside the breast contour to ensure target dosage due to the lack of buildup and lateral scatter [20-21]. Our proposed hybrid VMAT plans provide better target coverage and dose homogeneity than that of the 3D-CRT plans, and proved to be a safe technique upon evaluating the toxicity [18]. However, in our method, the dose distribution in the junction region may be affected by patient motion.

Patient motion can affect the accuracy of dose delivery and is significant for achieving a successful treatment. Field edges with a steep dose gradient may have a significant impact on the dose distribution in the junction region [6-8]. A perfect alignment in the junction region between adjacent fields is difficult to accomplish even when a phantom is used. A robust technique in the junction of adjacent treatment fields should therefore be considered to avoid the emergence of hot and cold spots. The largest concern is the emergence of possible hot and cold spots in the junction region in case of patient motion between the 3D-CRT and VMAT plans. In our hybrid VMAT technique, the cranial side of the tangential field creates a dose gradient by closing the jaw which gradually decreases the delivered dose and results in minimizing hot and cold spots in the junction region. We therefore believe that our hybrid-VMAT technique is highly insensitive to patient motion.

The purpose of this study was to investigate the impact of patient motion between the 3D-CRT and VMAT plans on dose distribution for locoregional breast cancer patients undergoing supraclavicular node treatment. We created the dose gradient using different jaw settings for the 3D-CRT field on the cranial side. The robustness in the junction region was evaluated by simulating the isocenter shift.

\section{Material And Methods \\ 2.1 Patient}

This study was approved by the Institutional Review Board of Hiroshima University (E-947). Ten postmastectomy breast cancer patients with supraclavicular nodes who received adjuvant loco-regional RT at our institution between August 2020 and September 2020 were selected for this dosimetric comparison 
study. Patients were immobilized in customized vacuum bags in the supine position with arms raised above their head for the ease of treatment position reproducibility. A radiopaque wire was placed on the patient midline and mid-axillary line by a radiation oncologist during simulation to better define the clinical target volume (CTV) extension. Computed tomography (CT) images for treatment planning were acquired with a $2.5 \mathrm{~mm}$ slice thickness (580W, GE Healthcare, Milwaukee, WI, USA) under free breathing. All CT data were exported to the Eclipse (version 13.5, Varian Medical Systems, Palo Alto, CA, USA) treatment planning system (TPS) for target and organs at risk (OAR) delineation and treatment planning.

\subsection{Target and OARs delineations}

The CTV contoured the entire ipsilateral chest wall area with supraclavicular nodes based on the Radiation Therapy Oncology Group (RTOG) Breast Cancer Atlas and clinical data for each patient. The planning target volume (PTV) was created by adding a uniform margin of $5 \mathrm{~mm}$ around the CTV. The evaluation structure of the "PTV-modified" region was created by subtracting the PTV with a $2 \mathrm{~mm}$ margin on the skin surface and lung around the PTV. The bilateral lungs, heart, contralateral breast, ipsilateral humeral head, spinal cord, and esophagus were contoured as OARs. All delineation processes were checked by radiation oncologists.

The prescription dose was 50 Gy given in 25 fractions to the conserved breast or chest wall and regional lymph nodes using 6-MV photons with a maximum available dose rate of $600 \mathrm{MU} / \mathrm{min}$. Figures 1 and 2 present the procedure beam arrangement, and an example of the coronal view of the dose distribution on the "3D-CRT plan on chest wall area", "VMAT plan on supraclavicular nodes", and plan sum. First, the isocenter was placed $2 \mathrm{~cm}$ caudal to the upper sternum. The gantry angles and field settings of the mediolateral $(\mathrm{ML})$ field were determined while considering the dose to the lung and heart. The opposing lateromedial (LM) field was created to have the beam divergence match the $\mathrm{ML}$ field. The multileaf collimator (MLC) leaves were opened anteriorly at least $2 \mathrm{~cm}$ to ensure coverage of the breast in case of breathing and swelling. The MLCs were adjusted to maximize shielding of the heart and lungs. The fieldin-field technique used three individual fields with the jaw shifts to create the dose gradient (Fig. 1-a), and the tangential fields for the chest wall area were calculated. The weight of these segments was adjusted to be approximately equal. The tangential field was normalized at the maximum dose $\left(D_{\max }\right)$ in the chest wall area. A marginal zone with an insufficient dose ( $\leq 95 \%$ of prescription dose) on the tangential irradiation was extracted and was used for the VMAT optimized structure to cover the PTV dose. In this study, two plans were created for each patient, with or without the jaw shifted on the cranial side of the 3D-CRT plan, which were defined as "low dose gradient" and "high dose gradient".

Second, the VMAT plan created two coplanar arcs (one counterclockwise and another clockwise) with gantry rotation angles of $240^{\circ}$ (ranging from $60^{\circ}$ to $181^{\circ}$ and $181^{\circ}$ to $60^{\circ}$ for the right side and from $179^{\circ}$ to $300^{\circ}$ and $300^{\circ}$ to $179^{\circ}$ for the left side), with the collimator angle of each arc set to $10^{\circ}$ or $80^{\circ}$ to avoid a tongue-and-groove effect at the same isocenter (Fig. 1-b). One arc with collimator angle of 80 only covered for the supraclavicular area due to max leaf travel. The VMAT plan for the supraclavicular area and marginal zone were optimized to the whole PTV the based on the results of the calculation of the tangential fields for the chest wall area. A ring structure was used to control high-dose areas 
surrounding the PTV by assigning maximum dose objectives. Additional avoidance structures were used to reduce dose to the lung, in particularly, for low dose. The planning objectives for the VMAT plan were the same for the comparison plans.

Finally, hybrid plans were created by the sum of the 3D-CRT and VMAT plans (Fig. 2). Cumulative dose volume histograms (DVHs) were evaluated to assess target volumes and OARs. The details of the PTV and OAR dose constraints are shown in Table 1. Figure 3 presents the dose profile in the junction region, where each plan indicates the slope shape, while the summation of the two plans is approximately homogeneous. In this study, we defined the treatment plan as a "nominal plan" The dose distributions and DVHs of the hybrid VMAT plans with high or low dose gradients in the 3D-CRT plan were evaluated and compared. A final dose calculation was performed using the Acuros XB (AXB) with a dose grid matrix of $2.5 \mathrm{~mm}$.

Table 1

Dose constraints for Hybrid VMAT optimization

\begin{tabular}{|llll|}
\hline & Index & Constraint & Preferable \\
\hline PTV-modified & $\mathrm{D}_{95 \%}(\%)$ & $92 \%-96 \%$ & \\
& Max $(\mathrm{Gy})$ & $\leqq 115 \%$ & $\leqq 120 \%$ \\
\hline & $\mathrm{D}_{50 \%}(\%)$ & $97-103 \%$ & \\
\hline Esophagus & $\mathrm{D}_{1 \mathrm{cc}}(\mathrm{Gy})$ & $\leqq 25 \mathrm{~Gy}$ & $\leqq 20 \mathrm{~Gy}$ \\
\hline & $\mathrm{D}_{5 \mathrm{cc}}(\mathrm{Gy})$ & $\leqq 20 \mathrm{~Gy}$ & $\leqq 15 \mathrm{~Gy}$ \\
\hline Lungs & $\mathrm{V}_{5 \mathrm{~Gy}}(\%)$ & $35 \%$ & $30 \%$ \\
& Mean $(\mathrm{Gy})$ & $\leqq 10 \mathrm{~Gy}$ & $\leqq 8 \mathrm{~Gy}$ \\
\hline
\end{tabular}

\subsection{Perturbed dose evaluation}

To investigate the impact of patient motion errors between the 3D-CRT and VMAT plans on the dose distribution, we simulated the robustness of the hybrid VMAT plan. To assess the potential risk associated with patient motion in the superior-inferior ( $\mathrm{SI}$ ) direction, the dose distribution of the VMAT plan was shifted by 1,2 , and $3 \mathrm{~mm}$ to the superior (separation) and inferior (overlap) directions. The summed dose distributions with all simulated patient motions were compared with the nominal dose distribution (no simulated patient motion errors). The shifted plans were recalculated with the same monitor units (MUs), gantry/collimator angles, and the MLC shape of the nominal plan. The $\mathrm{D}_{2 \%}$ and $\mathrm{D}_{98 \%}$ values were assessed as indicators of hot and cold spots, which are relevant for the overlap-and separation-simulated plans, respectively. 


\section{Results}

Comparison of treatment planning

The DVH statistics of the treatment plans of the examined patients are shown in Table 2. All hybrid VMAT plans resulted in a similar dose coverage to the PTV and OARs. All plans were clinically acceptable.

Table 2

Doses to the target and OARs using Hybrid VMAT with 3D-CRT on high or low dose gradient.

\begin{tabular}{|llll|}
\hline & Index & $\begin{array}{l}\text { Hybrid VMAT plan with high dose } \\
\text { gradient on 3D-CRT plan }\end{array}$ & $\begin{array}{l}\text { Hybrid VMAT plan with low dose } \\
\text { gradient on 3D-CRT plan }\end{array}$ \\
\hline $\begin{array}{l}\text { PTV- } \\
\text { modified }\end{array}$ & $\begin{array}{l}\mathrm{D}_{95 \%} \\
(\%)\end{array}$ & $95.8 \pm 1.0(94.3-97.9)$ & $95.7 \pm 0.9(94.4-97.1)$ \\
& $\begin{array}{l}\text { Max } \\
(\mathrm{Gy})\end{array}$ & $109.0 \pm 1.7(106.7-112.1)$ & $108.0 \pm 1.2(106.6-109.8)$ \\
\hline & $\begin{array}{l}\mathrm{D}_{50 \%} \\
(\%)\end{array}$ & $99.7 \pm 0.7(98.7-101.5)$ & $99.6 \pm 0.7(98.8-101.5)$ \\
\hline Esophagus & $\begin{array}{l}\mathrm{D}_{1 \mathrm{cc}} \\
(\mathrm{Gy})\end{array}$ & $16.8 \pm 2.0(13.4-20.4)$ & $16.4 \pm 1.8(12.8-19.1)$ \\
\hline & $\begin{array}{l}\mathrm{D}_{5 \mathrm{cc}} \\
(\mathrm{Gy})\end{array}$ & $11.4 \pm 1.8(8.6-13.9)$ & $11.5 \pm 2.1(8.2-14.0)$ \\
\hline Lungs & $\begin{array}{l}\mathrm{V}_{5 \mathrm{~Gy}} \\
(\%)\end{array}$ & $27.0 \pm 4.1(19.3-34.9)$ & $27.1 \pm 4.3(19.5-34.9)$ \\
\hline & $\begin{array}{l}\text { Mean } \\
(\mathrm{Gy})\end{array}$ & $7.7 \pm 1.4(5.7-10.0)$ & $7.7 \pm 1.5(5.7-10.0)$ \\
\hline Group averages with ranges shown in parentheses $(\mathrm{n}=10)$. & \\
\hline
\end{tabular}

Robustness evaluation

Figure 4 presents an example of the dose distribution generated with the hybrid VMAT plan with high - or low-dose gradients on the 3D-CRT plan. Compared to the hybrid VMAT plan with a high dose gradient in the 3D-CRT plan (Fig. 4-a), the hybrid VMAT plan with a low dose gradient on the 3D-CRT plan resulted in fewer hot and cold spots (Fig. 4-b). Figure 5 presents the dose profiles acquired in the junction region. Compared to the nominal plan, the simulation of the $\pm 3 \mathrm{~mm}$ SI direction in the hybrid VMAT plan with a high dose gradient in the 3D-CRT plan resulted in an over (132.0\%) and under dose (72.0\%) in the junction region, as depicted in Fig. 5-a. For the hybrid VMAT plan with a low dose gradient on the 3D-CRT plan, the introduced shift resulted in a dose only slightly over $(115.0 \%)$ and under $(85.0 \%)$ in the junction region, as shown in Fig. 5-b. Figure 6 presents the DVH of the CTV for a patient with overlapped or separated shifted evaluations. The maximum or minimum dose to the CTV for the hybrid VMAT plan with a high dose gradient on the 3D-CRT plan apparently worsened, whereas the hybrid VMAT plan with a low dose 
gradient on the 3D-CRT plan appeared to be robust against patient motion. Figure 7 presents the CTV $D_{2} \%$ and $D_{98 \%}$ calculated to compare each of the six shifted plans to the nominal plan. The $D_{2 \%}$ to the CTV with perfectly aligned fields for the hybrid VMAT plan with high- or low-dose gradients on the 3D-CRT plan increased from $102.8 \% / 102.9-107.2 \% / 105.7 \%, 114.9 \% / 110.9 \%$, and $122.2 \% / 115.5 \%$ for each $1 \mathrm{~mm}, 2$ $\mathrm{mm}$, and $3 \mathrm{~mm}$ overlapped shift, respectively (Fig. 7-a). The $\mathrm{D}_{98 \%}$ to the CTV with perfectly aligned fields for the hybrid VMAT plan with high - or low-dose gradients on the 3D-CRT plan decreased from 95.7$95.6 \%$ to $90.0 \% / 93.1 \%, 81.2 \% / 88.4 \%$, and $72.8 \% / 83.5 \%$ for each $1 \mathrm{~mm}, 2 \mathrm{~mm}$, and $3 \mathrm{~mm}$ separated shift, respectively (Fig. 7-b). Dose differences due to patient motion can be decreased using our hybrid VMAT technique that utilizes a low dose gradient on the 3D-CRT plan.

\section{Discussion}

The hot and cold spots in the junction region are one of the concerns for breast cancer RT with supraclavicular nodes. With conventional radiotherapy techniques using two opposing tangential and anterior fields, it is extremely difficult to deliver a homogeneous dose to the PTV in the junction region. In addition, this conventional radiotherapy technique is sensitive to possible misalignment errors and causes the emergence of hot and cold spots in the junction region [6]. The effect of the matching techniques on the dose variations in the junction region was investigated using a breast phantom [6]. Two different treatment techniques were compared under an overlap of $5 \mathrm{~mm}$, a separation of $5 \mathrm{~mm}$, and it was concluded that set-up difficulties influence the dose distribution. The conventional 3D-CRT treatment plan has a high dose gradient and the junction region is easily over or underdosed with the introduction of mechanical inaccuracies or possible patient movement during the treatment. The effect of uncertainty in the jaw positioning on dose distribution in the junction region was investigated [7]. The jaw positional errors should be managed to be less than $1 \mathrm{~mm}$ according to the American Association of Physicists in MedicineTask Group 40 [22].

A combination of two tangential fields and VMAT for breast cancer has already been reported [15]. Their strategy includes 2 tangential open fields with a $2 \mathrm{~cm}$ cranial slip zone delivering $85 \%$ of the chest wall area. In this study, we investigated the robustness of our proposed hybrid VMAT in breast cancer to achieve dose homogeneity in the junction region. Our proposed hybrid VMAT technique with a low dose gradient on the 3D-CRT plan could reduce the over and under dosage in the junction region. As shown in the dose profile, extreme patient motion $( \pm 3 \mathrm{~mm})$ can potentially lead to areas of high and low doses that can be up to approximately $30 \%$ different from the nominal plan. On average, the $\mathrm{D}_{2 \%}$ to the CTV of $1.5 \%$, $3.5 \%, 5.5 \%$, and $\mathrm{D}_{98 \%}$ to the CTV of $3.3 \%, 8.2 \%$, and $12.8 \%$ for the $1 \mathrm{~mm}, 2 \mathrm{~mm}$, and $3 \mathrm{~mm}$, overlapped/separated shifted evaluations were improved, respectively. This is because a shifting jaw on the 3D-CRT plan will result in a low dose gradient from the VMAT also being smooth in the junction region.

Patient setup and motion errors during treatment can result in small shifts in treatment fields; typical misalignment errors may vary between $0 \mathrm{~mm}$ and $3 \mathrm{~mm}[23,24]$. As shown in the dose profile, our study 
demonstrated that a $3 \mathrm{~mm}$ patient motion in the SI direction resulted in a dose difference of approximately $15 \%$ in the junction region, using an even hybrid VMAT plan with a low dose gradient in the 3D-CRT plan. Daily patient setup and patient motion are random sources of errors throughout the entire treatment. Intrafraction motion is significantly limited with a translational median of $1.1 \mathrm{~mm}$ from the isocenter [24]. Patients are not likely to move in the SI direction (mean $=0.09 \pm 0.81 \mathrm{~mm}$, median $=0.04$ $\mathrm{mm}$ ) [23]. Patient monitoring with a surface scanning system is expected to decrease patient motion during treatment and decrease the effect of field separation or overlap. The immobilization positioning system is also essential to minimize patient motion during the time of treatment. Studies have reported significantly improved reproducibility and decreased random and systematic inter-fraction errors, with the use of an immobilization device $[25,26]$. Some patients feel comfortable positioning themselves during treatment, as indicated with a simulation using an immobilization device [27]. The beam-on-time of our hybrid VMAT approach for breast cancer patients is required for only approximately 2-3 min after image guidance. Therefore, we believe that the patient motion with the immobilization device and our proposed hybrid VMAT is minimal during treatment.

A limitation of this study was that we only examined one dose gradient with a jaw shift. A large jaw shift contributes to a shallow dose gradient and a more robust plan for patient motion. The low-dose gradient plan was able to decrease the dose differences when patient shift errors were introduced, as was also found for the dose profiles, by a maximum of $12.8 \%$ for the $3 \mathrm{~mm}$ shift. However, a greater dose contribution to the lung on VMAT should be considered for respiratory motion and organ deformation [19]. A balance between the junction dose step size length and dose step size should be considered based on plan quality. The issue of dose junction associated with large treatment volumes presents problems with other sites, such as craniospinal irradiation and total marrow irradiation [28-30]. In these treatments, the couch is moved next to the treatment plan position because of the restricted field size. Therefore, a shallow dose gradient is used for plan robustness due to set-up error and patient motion at these treatment sites. It should be stressed that this study considered a maximum dose variation in the worst scenario. Intrafraction respiratory chest wall motion and the daily set-up errors have blurred the cold spots or hot spots in the junction region. Mathematical calculations of the probability distribution should be considered for the number of fractions because day-to-day setup variations were random.

\section{Conclusions}

Our study demonstrated that patient motion in the SI direction significantly affects the accuracy of breast cancer treatment in the junction region using hybrid VMAT. Hybrid VMAT with a low-dose gradient on the 3D-CRT plan can produce a comparable patient plan while significantly reducing the magnitude of any over- or under-dosing that may occur from patient motion. A more robust and accurate treatment delivery could be achieved without considering the breathing motion for breast cancer treatment using our proposed hybrid VMAT technique.

\section{Declarations}




\section{Conflict of interest}

The authors declare that they have no conflict of interest to disclose.

Presentation: None

Fund: None

\section{References}

1. Veronesi U, Cascinelli N, Mariani L et al (2002) Twenty-year follow-up of a randomized study comparing breast-conserving surgery with radical mastectomy for early breast cancer. New Engl $\mathrm{J}$ Med 347:1227-1232

2. Fisher B, Anderson S, Bryant $\mathrm{J}$ et al (2002) Twenty-year follow-up of a randomized trial comparing total mastectomy, lumpectomy, and lumpectomy plus irradiation for the treatment of invasive breast cancer. N Engl J Med 347:1233-1241

3. Ragaz J, Olivotto IA, Spinelli JJ et al (2005) Locoregional radiation therapy in patients with high-risk breast cancer receiving adjuvant chemotherapy: 20-year results of the British Columbia randomized trial. J Natl Cancer Inst 97:116-126

4. Klein EE, Taylor M, Michaletz-Lorenz $\mathrm{M}$ et al (1994) A mono isocentric technique for breast and regional nodal therapy using dual asymmetric jaws. Int J Radiat Oncol Biol Phys 28:753-760

5. Miles EA, Venables K, Hoskin PJ, Aird EG, START Trial Management Group (2009) Dosimetry and field matching for radiotherapy to the breast and supraclavicular fossa. Radiother Oncol 91:42-48

6. Idzes MH, Holmberg O, Mijnheer BJ et al (1998) Effect of set-up uncertainties on the dose distribution in the match region of supraclavicular and tangential breast fields. Radiother Oncol 46:91-98

7. Hedin E, Bäck A, Chakarova R (2015) Jaw position uncertainty and adjacent fields in breast cancer radiotherapy. J Appl Clin Med Phys 16:240-251

8. Homann KL, Gates BE, Salehpour M et al (2010) Use of a matchline dosimetry analysis tool (MDAT) to quantify dose homogeneity in the region between abutting tangential and supraclavicular radiation fields. J Appl Clin Med Phys 11:3294

9. Balaji K, Yadav P, BalajiSubramanian S et al (2018) Hybrid volumetric modulated arc therapy for chest wall irradiation: For a good plan, get the right mixture. Phys Med 52:86-92

10. Yao W (2013) A two-point scheme for optimal breast IMRT treatment planning. J Appl Clin Med Phys $14: 4525$

11. Farace P, Zucca S, Solla I et al (2012) Planning hybrid intensity modulated radiation therapy for whole-breast irradiation. Int J Radiat Oncol Biol Phys 84:e115-e122

12. Xie $\mathrm{X}$, Ouyang $\mathrm{S}$, Wang $\mathrm{H}$ et al (2014) Dosimetric comparison of left-sided whole breast irradiation with 3D-CRT, IP-IMRT and hybrid IMRT. Oncol Rep 31:2195-2205 
13. Jeulink M, Dahele M, Meijnen P et al (2015) Is there a preferred IMRT technique for left-breast irradiation? J Appl Clin Med Phys 16:197-205

14. Lin J, Yeh D, Yeh H et al (2015) Dosimetric comparison of hybrid volumetric-modulated arc therapy, volumetric-modulated arc therapy and intensity-modulated radiation therapy for left-sided early breast cancer. Med Dosim 40:262-267

15. van Duren-Koopman MJ, Tol JP, Dahele M et al (2018) Personalized automated treatment planning for breast plus locoregional lymph nodes using Hybrid RapidArc. Pract Radiat Oncol 8:332-341

16. Mayo CS, Urie MM, Fitzgerald TJ (2005) Hybrid IMRT plans-concurrently treating conventional and IMRT beams for improved breast irradiation and reduced planning time. Int $\mathrm{J}$ Radiat Oncol Biol Phys 61:922-932

17. Popescu CC, Olivotto IA, Beckham WA et al (2010) Volumetric modulated arc therapy improves dosimetry and reduces treatment time compared to conventional intensity-modulated radiotherapy for locoregional radiotherapy of left-sided breast cancer and internal mammary nodes. Int $\mathrm{J}$ Radiat Oncol Biol Phys 76:287-295

18. Doi Y, Nakao M, Miura H et al (2020) Hybrid volumetric-modulated arc therapy for postoperative breast cancer including regional lymph nodes: the advantage of dosimetric data and safety of toxicities. J Radiat Res 61:747-754

19. Rossi M, Boman E, Skyttä T et al (2018) Dosimetric effects of anatomical deformations and positioning errors in VMAT breast radiotherapy. J Appl Clin Med Phys 19:506-516

20. Giorgia N, Antonella F, Alessandro C et al (2011) Planning strategies in volumetric modulated are therapy for breast. Med Phys 38:4025-4031

21. Virén T, Heikkilä J, Myllyoja K et al (2015) Tangential volumetric modulated arc therapy technique for left-sided breast cancer radiotherapy. Radiat Oncol 10:79

22. Kutcher GJ, Coia L, Gillin M et al (1994) Comprehensive QA for radiation oncology: report of AAPM Radiation Therapy Committee Task Group 40. Med Phys 21:581-618

23. Reitz D, Carl G, Schonecker S et al (2018) Real-time intra-fraction motion management in breast cancer radiotherapy: analysis of 2028 treatment sessions. Radiat Oncol 13:128

24. Hattel SH, Andersen PA, Wahlstedt IH et al (2019) Evaluation of setup and intrafraction motion for surface guided whole-breast cancer radiotherapy. J Appl Clin Med Phys 20:39-44

25. Mitine C, Dutreix A, Van der Schueren E (1991) Tangential breast irradiation: influence of technique of set-up on transfer errors and reproducibility. Radiother Oncol 22:308-310

26. Thilmann C, Adamietz IA, Saran F et al (1998) The use of a standardized positioning support cushion during daily routine of breast irradiation. Int J Radiat Oncol Biol Phys 41:459-463

27. Nalder CA, Bidmead AM, Mubata CD et al (2001) Influence of a vac-fix immobilization device on the accuracy of patient positioning during routine breast radiotherapy. Br J Radiol 74:249-254

28. Seppälä J, Kulmala J, Lindholm P et al (2010) A method to improve target dose homogeneity of craniospinal irradiation using dynamic split field IMRT. Radiother Oncol 96:209-215 
29. Fogliata A, Bergström S, Cafaro I et al (2011) Cranio-spinal irradiation with volumetric modulated arc therapy: a multi-institutional treatment experience. Radiother Oncol 99:79-85

30. Mancosu P, Navarria P, Castagna L et al (2013) Interplay effects between dose distribution quality and positioning accuracy in total marrow irradiation with volumetric modulated arc therapy. Med Phys 40:111713

\section{Figures}

(a)

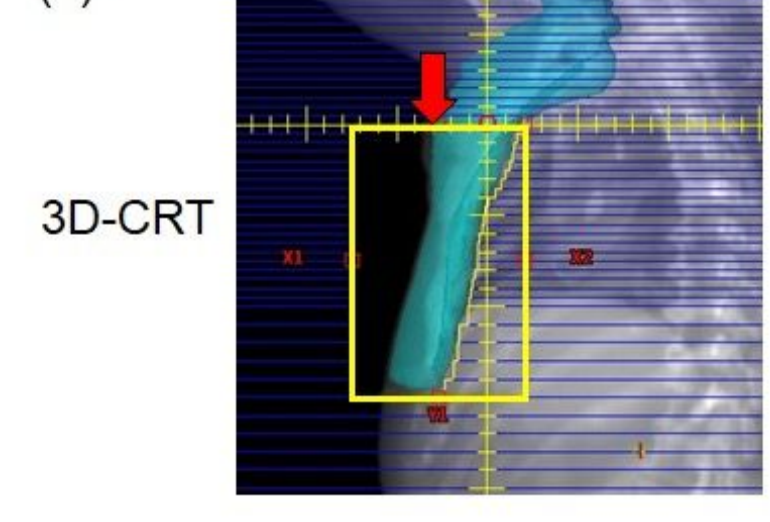

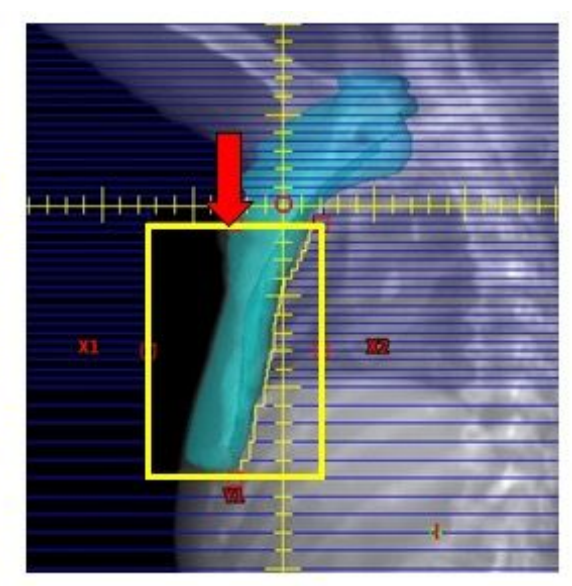

(b)
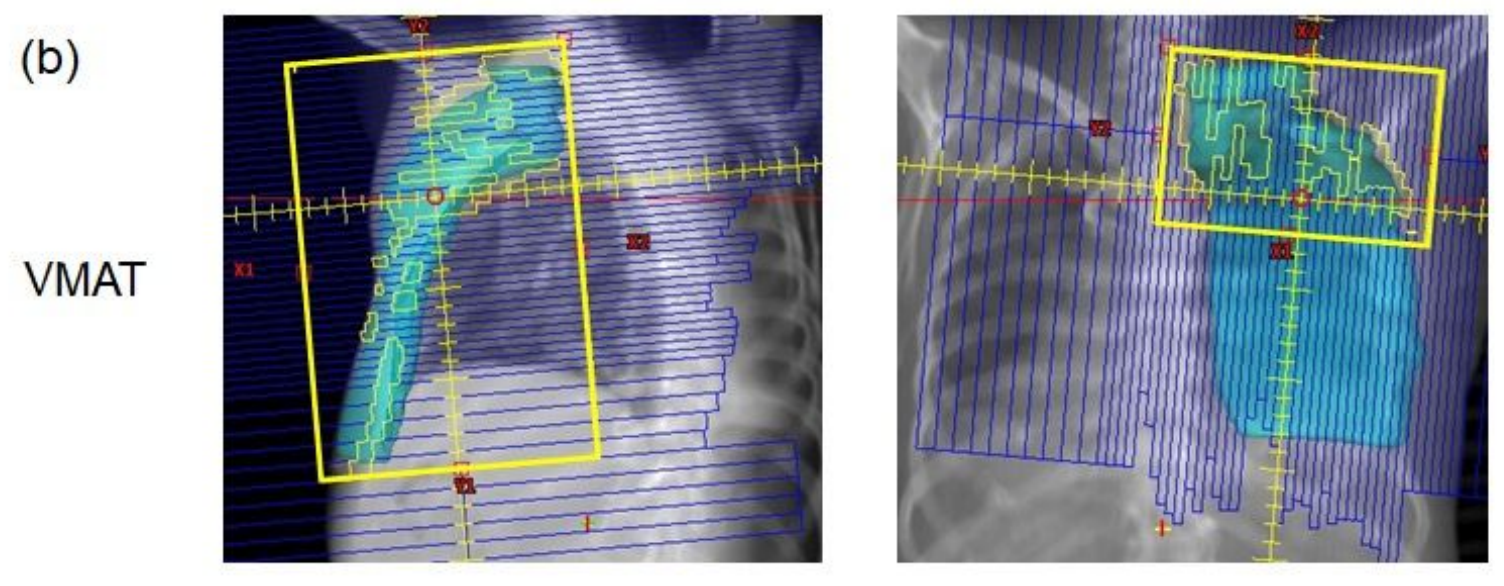

Figure 1

Typical field setup for the (a) 3D-CRT plan on chest wall area and (b) VMAT plan on supraclavicular area for a single right-sided patient. Dose gradient in the junction region was created by shifting the tangential field. The jaw is indicated by yellow. In segment 1 , the tangential field on the cranial side was fully open until the isocenter level. In segment 2 , the jaw moved $5 \mathrm{~mm}$ toward closure. In segment 3 , the jaw moved an additional $5 \mathrm{~mm}$ towards closure. The shifted jaw is indicated by the red arrow. For a better modulation, the jaw field size of the second arc was restricted in only the supraclavicular area. 


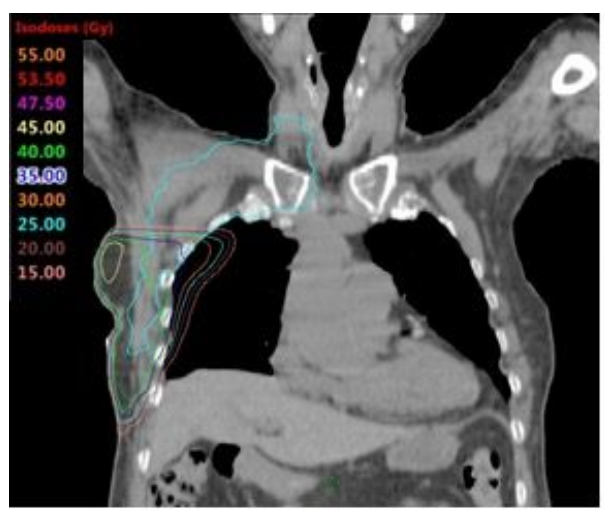

(a) 3D-CRT

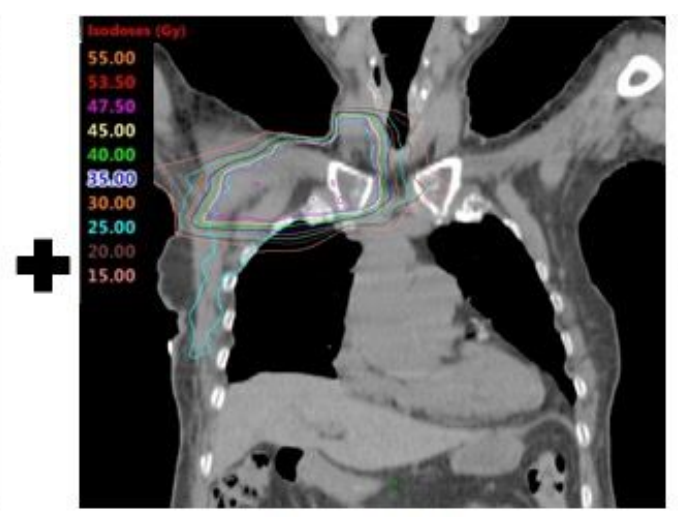

(b) VMAT

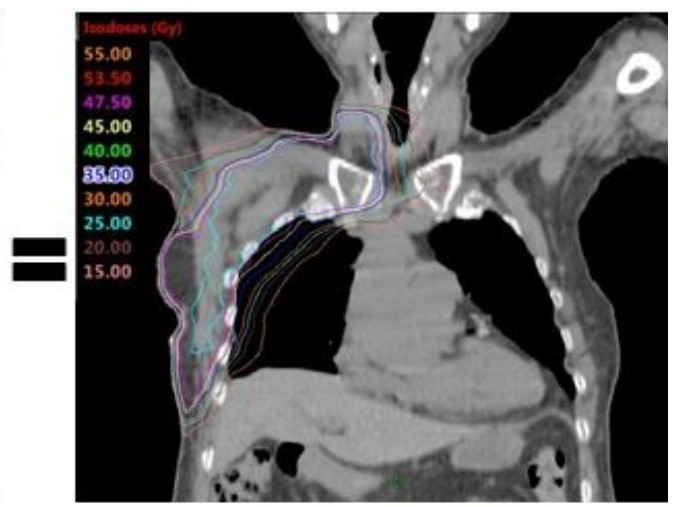

(c) Hybrid-VMAT

Figure 2

Procedure treatment planning (a) 3D-CRT plan on chest wall area, (b) "VMAT plan on supraclavicular nodes, and (c) Hybrid VMAT plan, which combined 3D-CRT and VMAT plans.

(a) High Dose gradient

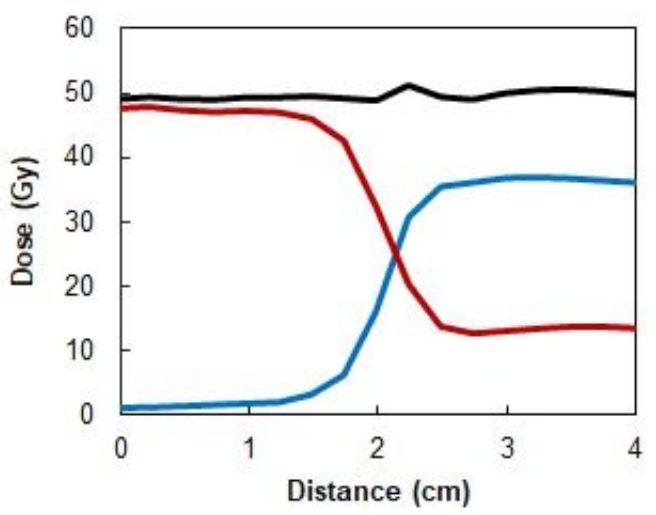

(b) Low dose gradient

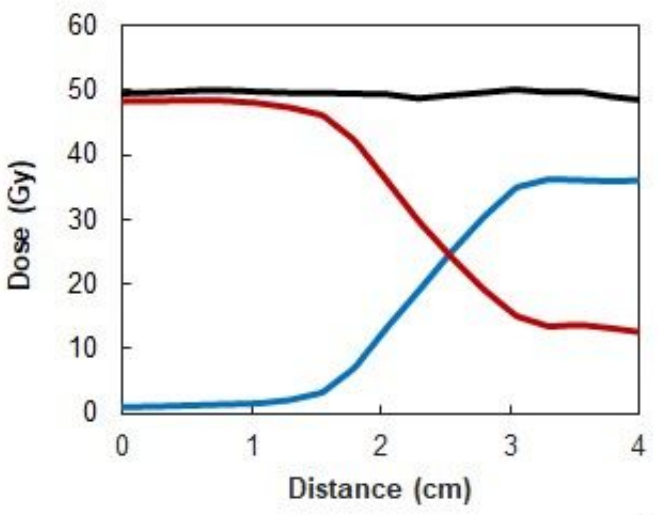

(c)

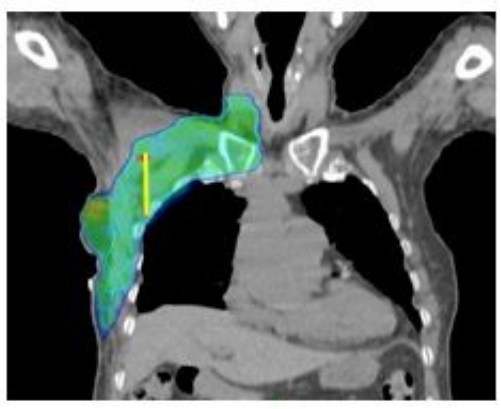

\section{Figure 3}

Dose profile for hybrid VMAT plan with (a) high or (b) low dose gradients on 3D-CRT plan along (c) yellow line between the same two points in the junction region. Contributions of the 3D-CRT plan, VMAT plan, and the total sum dose, are indicated by the blue, red and black, respectively. Dose gradient of 3D-CRT plan without and with jaw shifted plans have a slope of (a) $11.0 \% / \mathrm{mm}$ or (b) $5.8 \% / \mathrm{mm}$, respectively. 
(a) High dose gradient

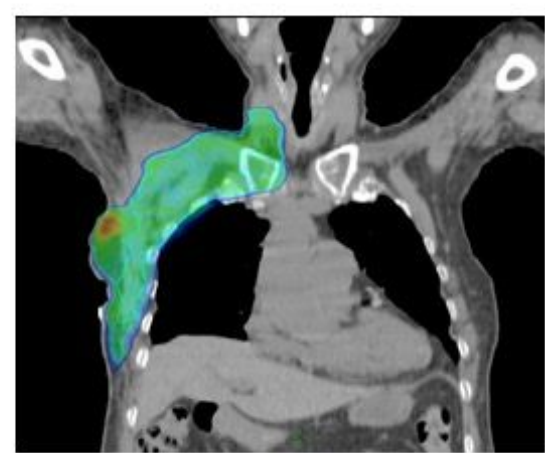

Nominal plan

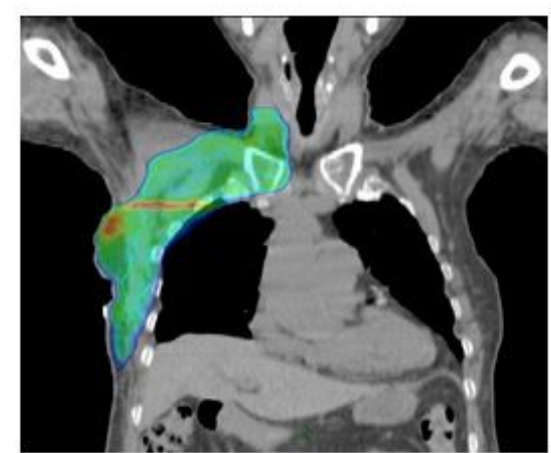

$+1 \mathrm{~mm}$ overlap

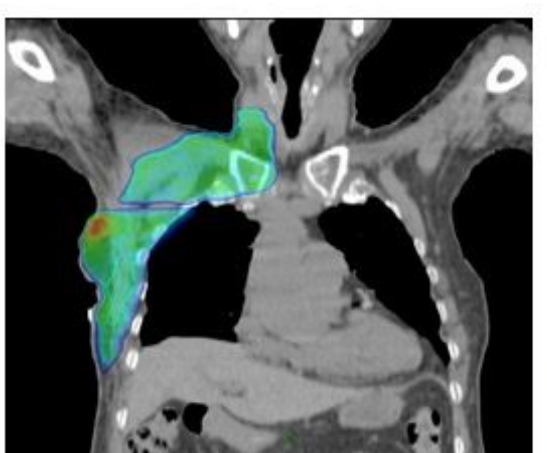

$-1 \mathrm{~mm}$ separation

(b) Low dose gradient

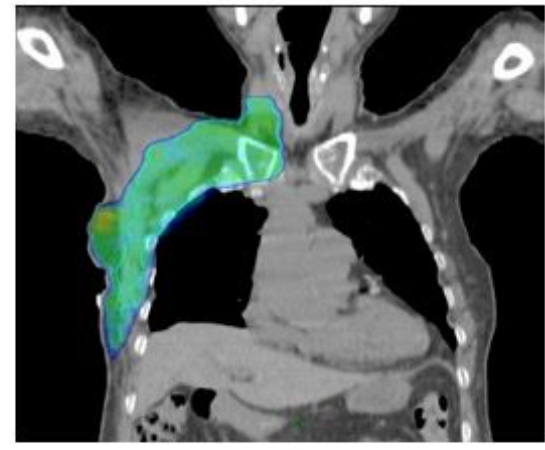

Nominal plan

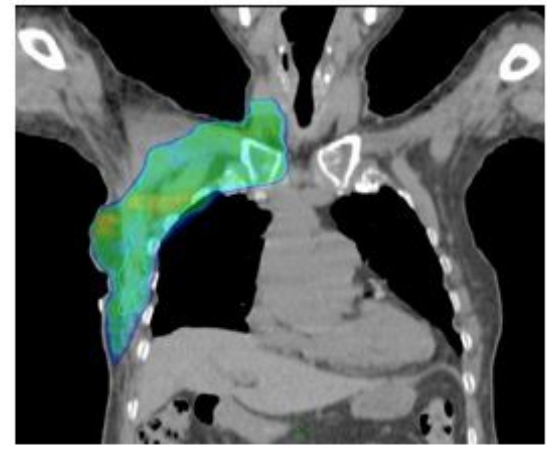

$+1 \mathrm{~mm}$ overlap

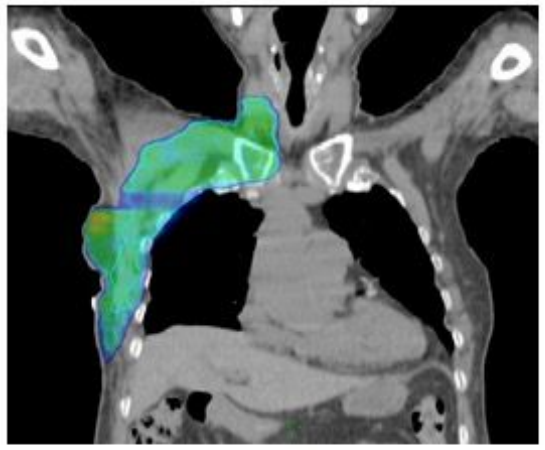

$-1 \mathrm{~mm}$ separation

Figure 4

An example of the dose distribution for (a) the hybrid VMAT plan with high dose gradient on 3D-CRT plan compared with (b) the hybrid VMAT plan with low dose gradient on 3D-CRT plan. Dose distribution in the coronal area illustrates the nominal (left panel), overlapped (center panel), and separated (right panel) evaluation plans. 
(a) High dose gradient

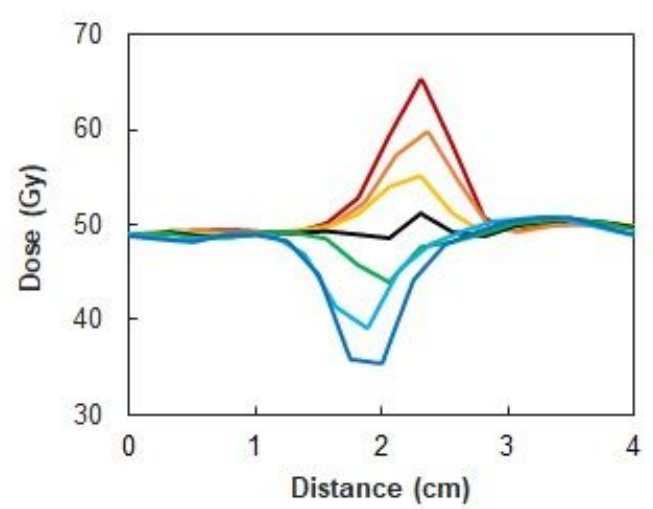

(b) Low dose gradient

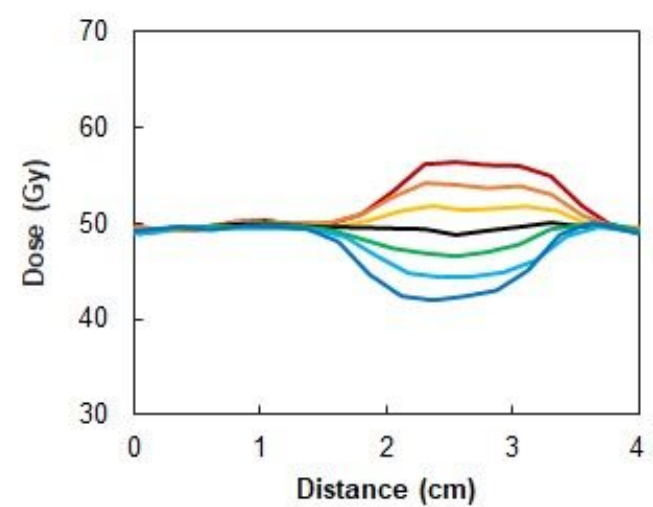

(c)

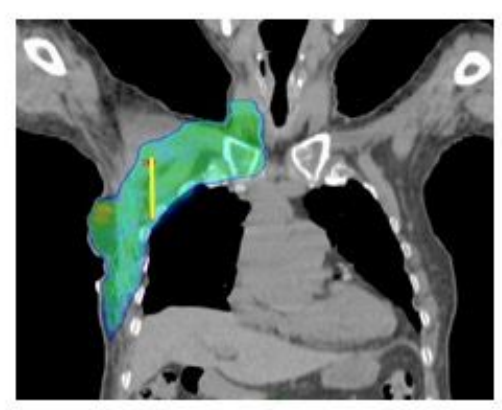

\section{Figure 5}

The dose profile of hybrid VMAT plan with (a) high or (b) low dose gradients on 3D-CRT plan for $1 \mathrm{~mm}, 2$ $\mathrm{mm}$, and $3 \mathrm{~mm}$ separated and overlapped shift along (c) yellow line in the junction region.

(a) High dose gradient

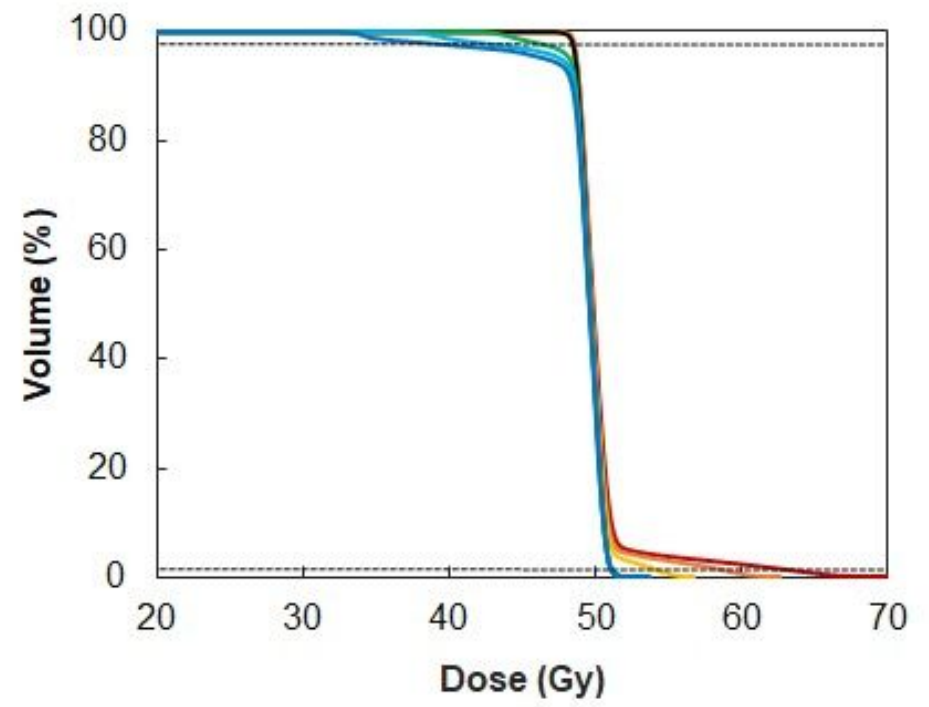

(b) Low dose gradient

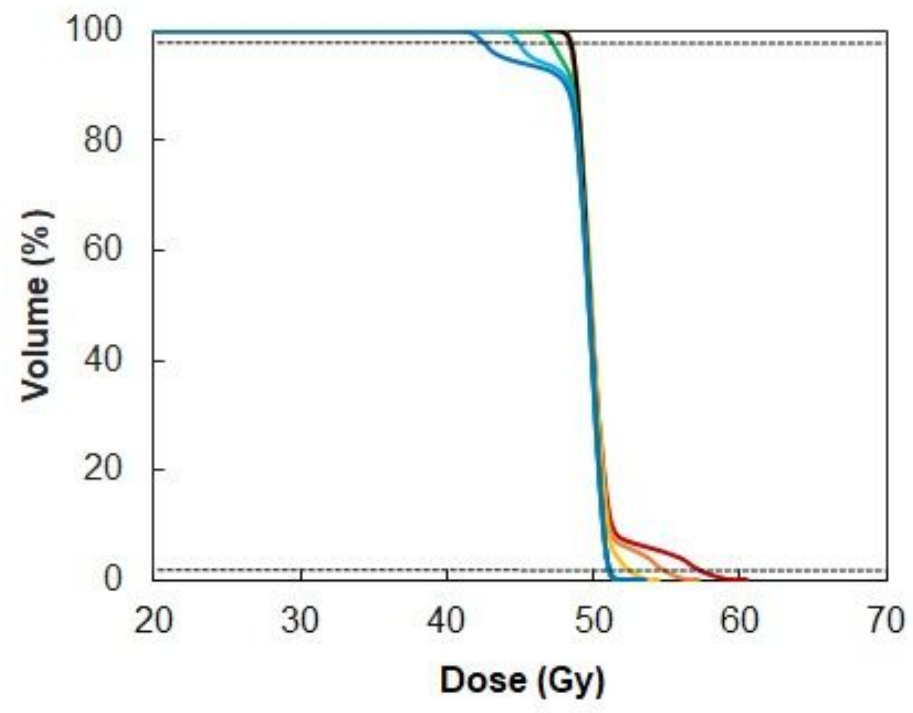

\begin{tabular}{|llll|}
\hline -overlap $3 \mathrm{~mm}$ & -overlap $2 \mathrm{~mm}$ & - overlap $1 \mathrm{~mm}$ & -nominal plan \\
- separation $1 \mathrm{~mm}$ & - separation $2 \mathrm{~mm}$ & - separation $3 \mathrm{~mm}$ & \\
\hline
\end{tabular}

Figure 6

Dose volume histograms (DVHs) of the clinical target volume (CTV) for hybrid VMAT plan with (a) high or (b) low dose gradients on 3D-CRT plan with $1 \mathrm{~mm}, 2 \mathrm{~mm}$, and $3 \mathrm{~mm}$ separated and overlapped shifts, respectively. Horizontal dash lines indicate D $98 \%$ and D2\%, respectively. 
(a)

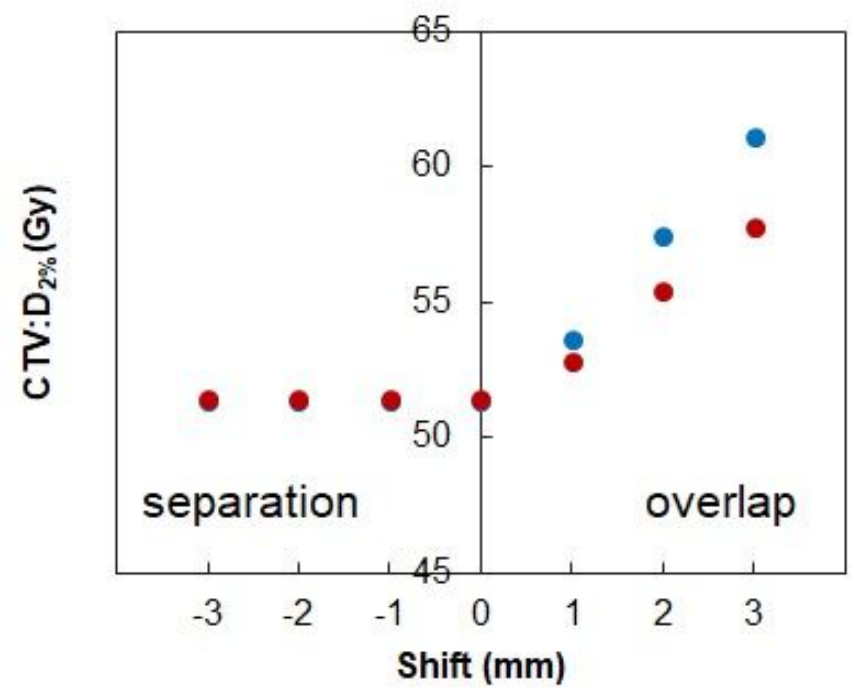

- High dose gradient (b)

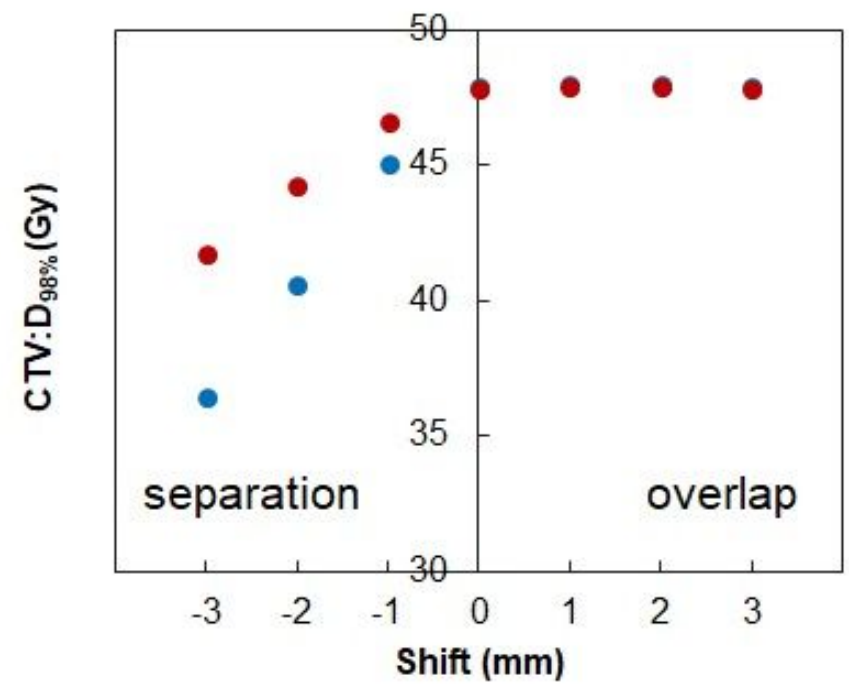

- Low dose gradient

\section{Figure 7}

Plot of average (a) D2\% and (b) D98\% dose to the CTV as a function of the simulated patient motion using the hybrid VMAT plan with high or low dose gradients on the 3D-CRT plan in ten patients. 